\title{
CMRR Boosted Instrumentation Amplifier for Biomedical Application
}

\author{
Ms. Amruta Bijwar \\ Research Scholar, Department of Instrumentation Engineering Amravati \\ amrutabijwar@gmail.com
}

\begin{tabular}{|l|l|}
\hline Article History & \multicolumn{1}{|c|}{ Abstract } \\
Article Submission & This research paper discusses about a design of an amplifier for its use in an Analog \\
27 September 2013 & Front End for Biomedical signal acquisition. The design of an AFE is also specific to \\
Revised Submission & the signal of interest. This paper deals with the design of an Analog Front End using \\
27 October 2013 & 180nm process. An amplifier is a key component of an AFE. For instrumentation \\
Article Accepted & amplifier to satisfy theoretical results the OPAMP used must be close to ideal. The \\
25 November 2013 & simulations are performed using TANNER EDA tool. \\
Article Published & Keywords: AFE, Voltage Transfer Characteristics, 180nm process, folded cascade \\
& OPAMP, Instrumentation Amplifier
\end{tabular}

\section{Introduction}

The Analog Front End is the most important part of a Signal Processing System. For a signal to be processed in any form say digital or analog, first it needs to be acquired properly in desired form. An Analog Front End makes way for this. The design of an AFE depends on the nature of signal of interest. Based on the nature the type of amplifier and filter that needs to be employed can be decided. The design also depends on various factors like controllability that needs to be offered, power, etc. By controllability, it means that the system can be programmable or specific to a particular biomedical signal say ECG, EEG or EMG.

As Shown in figure.1, an AFE basically consists of a pre amplifier, filter and a post amplifier. This is a conventional way of designing an AFE. Many contemporary methods are also available, few of which will be discussed in the forthcoming chapters. The objective of this system is to boost the signal at desired frequency and extracting it for further processing. The nature of the signal must remain the same at the same time the system must be able to sense even a signal with very small amplitude and also the selectivity of the filters must be good. There are many factors that need to be considered before getting into the design.

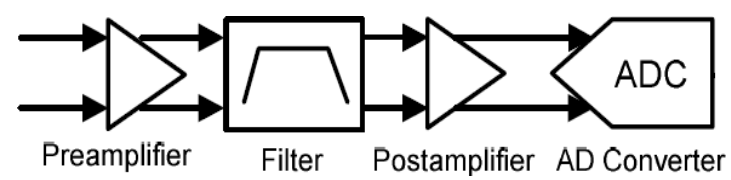

Fig. 1: Conventional Analog Front End

\section{Proposed Design of Folded Cascoded OPAMP}

The schematic of the folded cascode OPAMP designed is as shown in Figure 2. The load is comprised of a series of current mirrors that provides high output impedance. PMOS transistors are used at the input to avoid flicker noise. The folded cascode structure is usually implemented in a single stage. As in case of a basic two stage OPAMP there is no capacitance that is explicitly included to have a control over the frequency response. It is the parasitic junction capacitance that influences the response. 


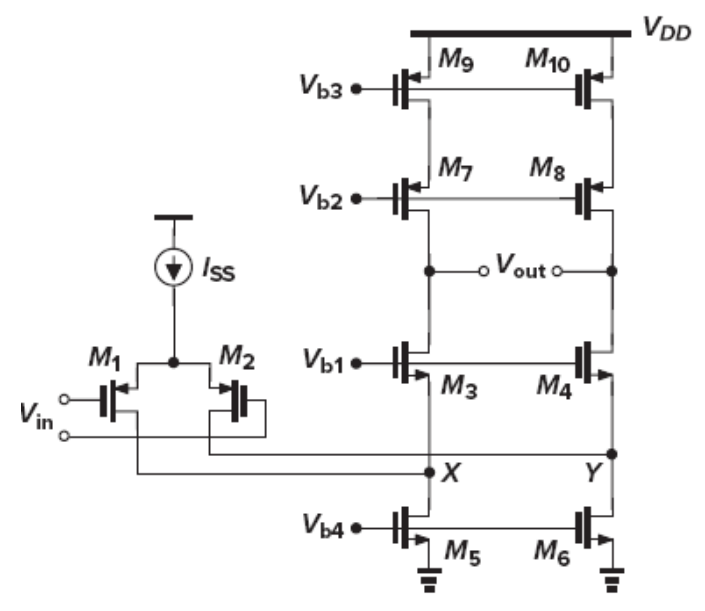

Fig. 2: Proposed folded cascoded op-amp design

The main advantage of this architecture is that the output swing is increased. The schematic representation of the folded cascade operational amplifier is shown in figure 3.

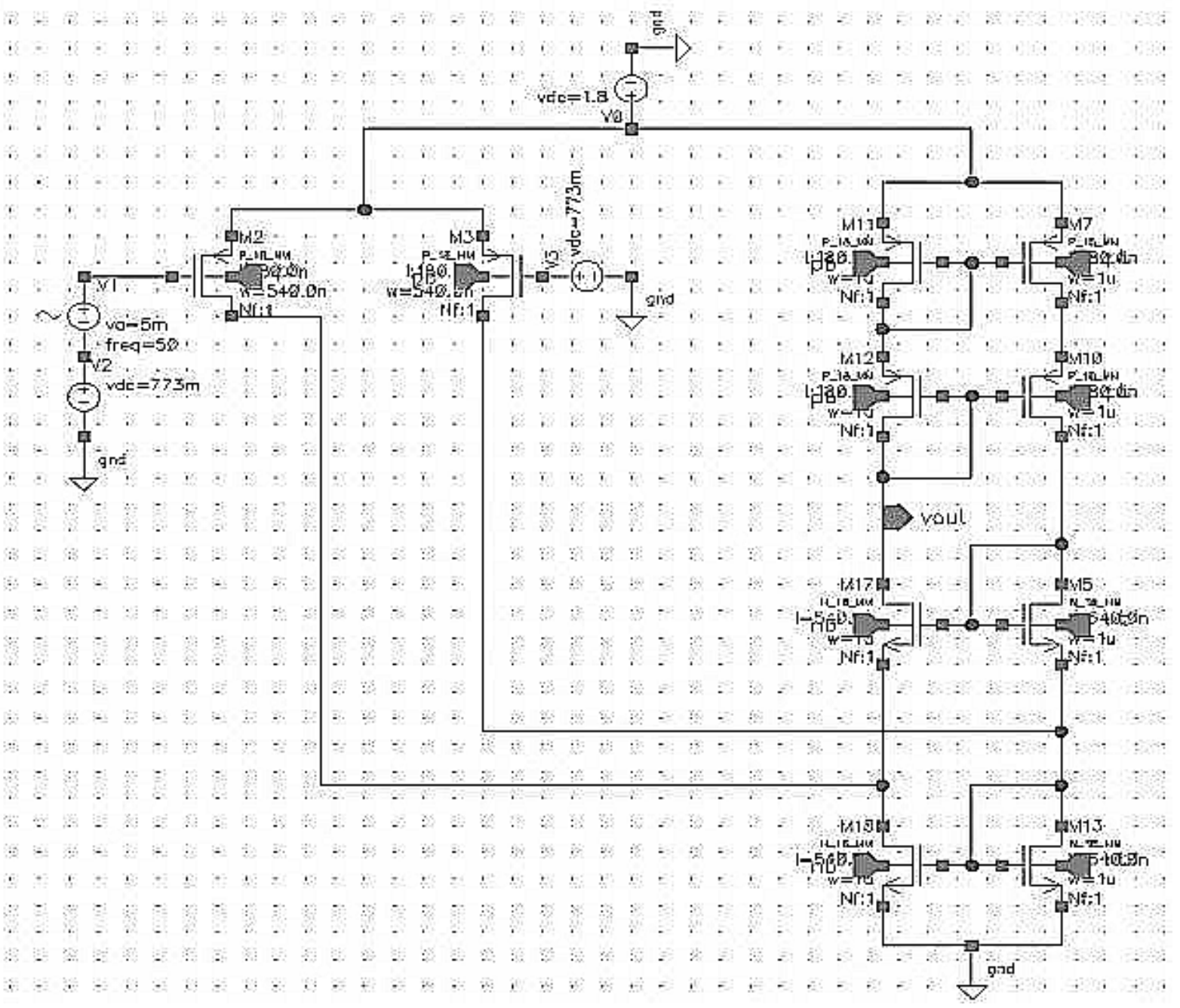

Fig. 3. Schemetic of the Folded Cascode OPAMP 
The sizes of the transistors play a vital role in this design. Various things like bias voltages, VTC of the amplifier, etc. depend directly or indirectly on the size of these transistors.

Table I. Transistor Sizing parameters

\begin{tabular}{|c|c|c|}
\hline \multicolumn{3}{|l|}{ Table Column Head } \\
\hline Transistor & Length & Width \\
\hline M2,M3 & $180 \mathrm{~nm}$ & 540nm \\
\hline M5,M7,M10,M11 & $540 \mathrm{~nm}$ & $1 \mathrm{um}$ \\
\hline M13,M18,M12,M17 & $540 \mathrm{~nm}$ & 1um \\
\hline
\end{tabular}

The AC Response of the designed Folded Cascode OPAMP is shown in Fig. 4. As it can be seen the gain of the OPAMP is $53.34 \mathrm{~dB}$.

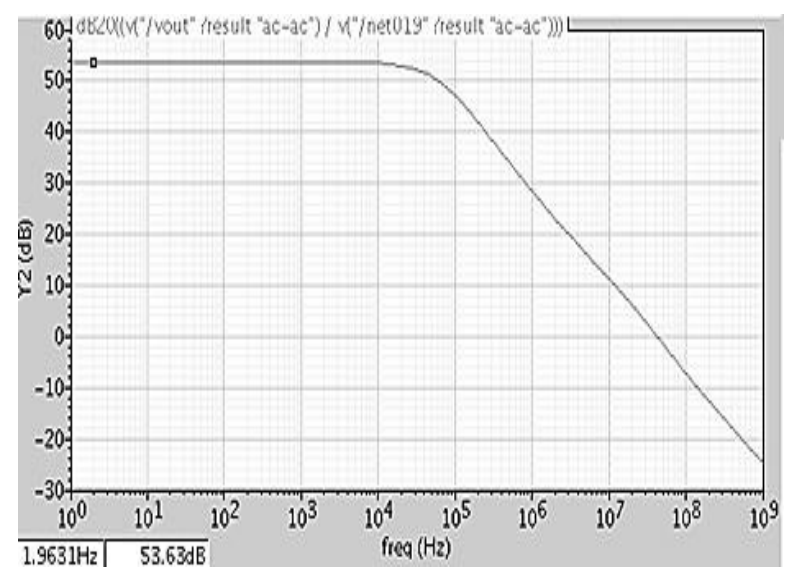

Fig. 4. AC Response of the Folded Cascode OPAMP

\section{Implementation of Proposed Design of Folded Cascoded OPAMP in Instrumentation Amplifier}

The low Ri hindrance of the instrumentation amplifier can be sorted out by using buffers made up of voltage followers in the input terminals. A voltage follower is connected in the input terminals of the differential amplifier.

The output is given by,

$$
\mathbf{V}_{\mathbf{o}}=\left(\mathbf{R}_{\mathbf{4}} / \mathbf{R}_{\mathbf{3}}\right)\left(\mathbf{1}+\mathbf{R}_{2} / \mathbf{R}_{1}\right)
$$

It can be seen that if the open loop gain of the OPAMP is high then the gain of the Instrumentation amplifier depends only on the passive components i.e. the resistors. The structure of the instrumentation amplifier is shown in figure 5 . 


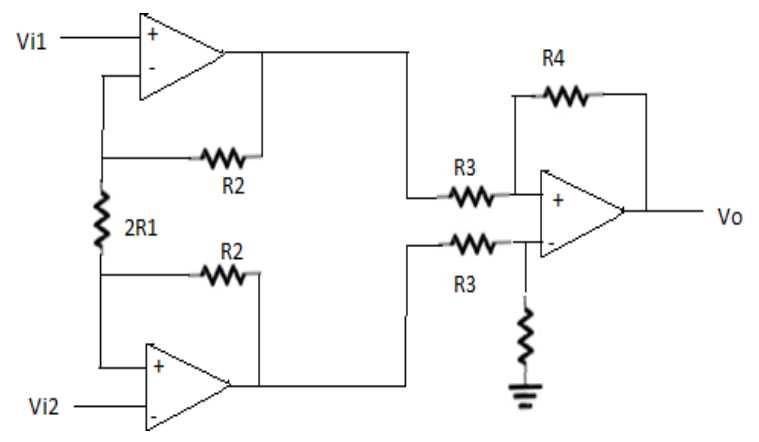

Fig. 5. Instrumentation Amplifier

The Figure 6 shows instrumentation amplifier that utilizes the Two Stage OPAMP previously designed. As mentioned earlier the input impedance of this amplifier is quite high compared to a single OPAMP. If the open gain of the OPAMP is made high then the Instrumentation Amplifier will have a response close to predicted. The OPAMPS in the first stage are use in non-inverting configuration while the one that is used in second stage is a difference amplifier.

In practical the resistors will be replaced by transistors and will be forced to operate in a specific region so that the resistance offered will remain the same throughout the range of the input.

One of the main disadvantages of this architecture is it is hard to maintain the resistances used for a wide range of output even if the resistors are replaced with transistors.

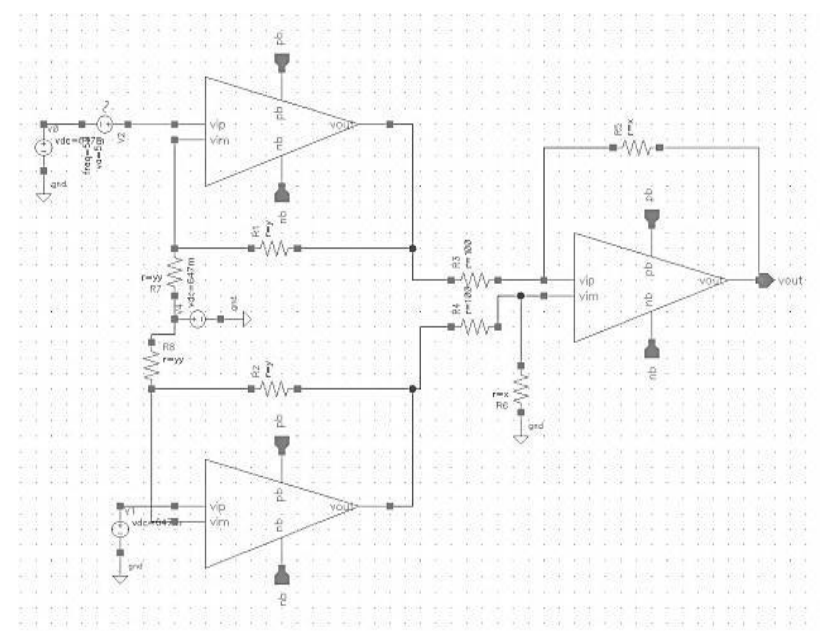

Fig. 6. Simulation of Instrumentation Amplifier with folded cascade op-amp

The main parameters of an OPAMP i.e. the common mode gain and differential mode gain are shown in this section. It can be seen that the same is around $-26 \mathrm{~dB}$ for a wide range of frequency. The differential mode gain should be as high as possible so that the OPAMP is close to an ideal one The common mode gain and differential mode gain analysis is shown in figure 7 and 8 respectively. 


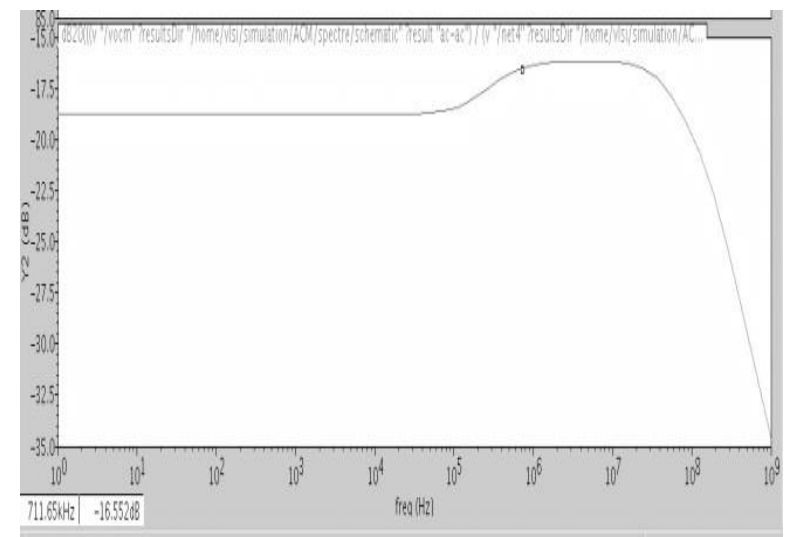

Fig. 6. Common Mode Gain of Instrumentation Amplifier with folded cascade op-amp

The differential mode gain of the OPAMP designed is $59 \mathrm{~dB}$ for a wide range of frequencies (till 1Mhz).Thus making it suitable for bio medical applications. From these two results the CMRR of the OPAMP can be found to be $79 \mathrm{~dB}$.

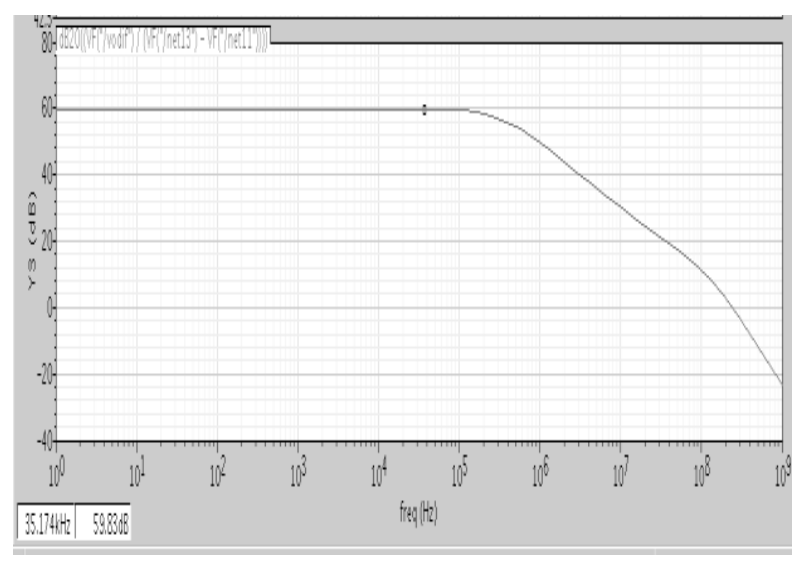

Fig. 7. Differential Mode Gain of Instrumentation Amplifier with folded cascade op-amp

The designed OPAMP has a high CMRR that makes it suitable for bio medical applications. As the gain and CMRR are high the gain of instrumentation amplifier designed from this OPAMP will be free from internal parameters and depend only on passive elements like resistors and capacitors. The simulation analysis of the proposed instrumentation amplifier is shown in figure 8 .

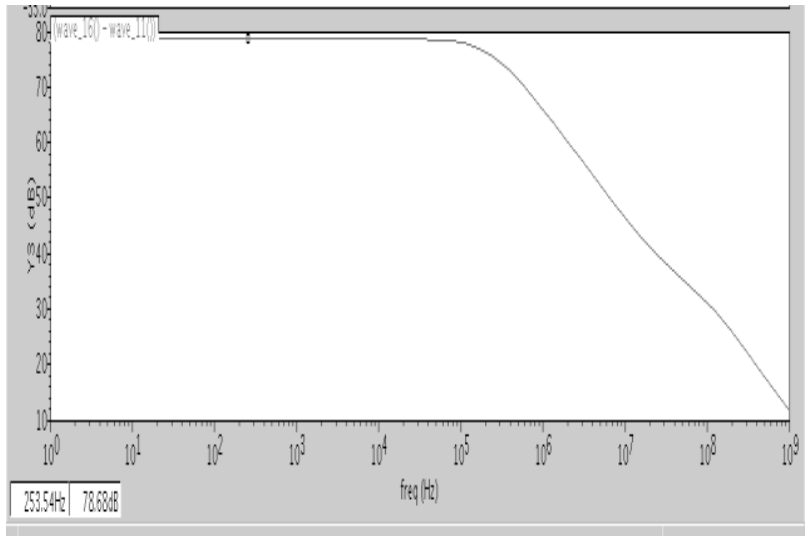

Fig. 8. CMRR of Instrumentation Amplifier with folded cascade op-amp 


\section{Conclusion}

The designed OPAMP has a high CMRR that makes it suitable for bio medical applications. As the gain and CMRR are high the gain of instrumentation amplifier designed from this OPAMP will be free from internal parameters and depend only on passive elements like the resistors and capacitors involved. The simulations are performed using TANNER EDA tool. Future works will be narrowed down toward implementation in Analog Front End.

\section{References}

[1] Mellita, Wei-Ming Chen, Wen-Chia Yang, Tzung-Yun Tsai, Herming Chiueh, Chung-Yu Wu, "The Design of CMOS General-Purpose Analog Front-End Circuit with Tunable Gain and Bandwidth for Biopotential Signal Recording Systems", 33rd Annual International Conference of the IEEE EMBS Boston, Massachusetts USA, August 30 - September 3, 2011.

[2] C.J. Deepu, X.Y. Xu, X.D. Zou, L.B. Yao, Y. Lian, “An ECG- on-Chip for Wearable Cardiac Monitoring Devices", 2010 Fifth IEEE International Symposium on Electronic Design, Test \& Applications.

[3] Andrea Abba, Antonio Manenti, Francesco Caponio, and Angelo Geraci, Senior Member, IEEE, "High Performance Analog Front-End for Digital Spectroscopy", IEEE transactions on nuclear science, Vol. 57, No. 4, August 2010.

[4] Jianbin Pan, Yuanfu Zhao, "Design of a Charge-Pump PLL for LVDS SerDes", Proceedings of international Multiconference of Engineers and Scientists, 2010, Vol II.

[5] Biman Chattopadhyay, Anant S Kamath, Gopalkrishna Nayak, "A 1.8GHz Digital PLL in 65nm CMOS”, 24th Annual Conference on VLSI Design, 2011.

[6] Limei Xiu, Zheying Li, "Design of a Low-power Programmable Gain Amplifier for Analog Front End Applications", Electric Information and Control Engineering (ICEICE), IEEE, April 15, 2011.

[7] N. V. Warke, P. P. Vaidya and J. M. Nair, "Exploring a new method for improving dynamic range of instrumentation amplifier for processing low voltage signals," 2013 International Conference on Emerging Trends in Communication, Control, Signal Processing and Computing Applications (C2SPCA), Bangalore, 2013, pp. 1-4.

[8] Goel and G. Singh, "Novel High Gain Low Noise CMOS Instrumentation Amplifier for Biomedical Applications," 2013 International Conference on Machine Intelligence and Research Advancement, Katra, 2013, pp. 392-396.

[9] S. Maréchal, O. Nys, F. Krummenacher, M. Chevroulet and M. Kayal, "A continuous-time instrumentation amplifier employing a novel auto-zeroing structure," Proceedings of the 20th International Conference Mixed Design of Integrated Circuits and Systems - MIXDES 2013, Gdynia, 2013, pp. 157-162.

[10] F. Del Cesta, A. N. Longhitano, P. Bruschi and R. Simmarano, "A wide input range instrumentation amplifier for impedance spectroscopy applications," Proceedings of the 2013 9th Conference on Ph.D. Research in Microelectronics and Electronics (PRIME), Villach, 2013, pp. 65-68.

[11] Prof. Naveen Jain. (2013). FPGA Implementation of Hardware Architecture for H264/AV Codec Standards. International Journal of New Practices in Management and Engineering, 2(01), 01 - 07. Retrieved from http://ijnpme.org/index.php/IJNPME/article/view/11

[12] Dr. Bhushan Bandre. (2013). Design and Analysis of Low Power Energy Efficient Braun Multiplier. International Journal of New Practices in Management and Engineering, 2(01), 08 - 16. Retrieved from http://ijnpme.org/index.php/IJNPME/article/view/12 